\title{
PREPARO CONVENCIONAL E CULTIVO MÍNIMO DO SOLO NA CULTURA DE MANDIOCA EM CONDIÇÕES DE ADUBAÇÃO VERDE COM ERVILHACA E AVEIA PRETA
}

\author{
DIFFERENCES BETWEEN CONVENCIONAL AND MINIMUM TILLAGE SOIL PREPARATION \\ FOR CASSAVA CROPPING WITH Vicia sativa AND Avena strigosa GREEN MANURE
}

\author{
Antonio Gabriel Filho ${ }^{1}$ Antonio Carlos dos Santos Pessoa ${ }^{1}$ Laércio Strohhaecker $^{2}$ \\ Jaime José Helmich ${ }^{2}$
}

RESUMO

Na cultura da mandioca, o preparo do solo é tradicionalmente realizado com uma aração e duas gradagens, o que pode causar compactação e erosão do solo e diminuição da produtividade. $O$ uso de plantas de cobertura do solo como adubação verde e o preparo do solo através do método de cultivo mínimo podem contribuir na diminuição do processo erosivo do solo e facilitar a colheita manual. Implantou-se o experimento em Latossolo Roxo eutrófico em Marechal Cândido Rondon (PR), visando à obtenção de dados sobre alterações em algumas propriedades físicas do solo, no esforço de arranquio e na produtividade de raízes de mandioca. $O$ delineamento experimental foi blocos ao acaso com oito tratamentos e quatro repetições, em esquema fatorial do tipo $2 x 4$, ou seja, dois sistemas de preparo do solo (cultivo mínimo e preparo convencional) e quatro tipos de cobertura vegetal do solo (aveia, ervilhaca, aveia em consórcio com ervilhaca e solo em pousio). Os resultados mostraram que a densidade do solo e a resistência do solo à penetração, na camada de 0 a $10 \mathrm{~cm}$, foram maiores nas áreas de preparo convencional do solo. Não houve diferença no esforço de arranquio e na produtividade de raízes de mandioca e cultivada com preparo convencional e cultivo mínimo do solo, tampouco das plantas de cobertura que antecederam o cultivo da mandioca Isso, possivelmente, ocorreu porque o solo apresentava-se com boa estrutura, com alta fertilidade natural e não houve deficiência hídrica na região durante o período de avaliação. Portanto, o cultivo mínimo pode substituir o preparo convencional do solo, nessas condições, diminuindo, assim, os custos de implantação da lavoura na cultura da mandioca, reduzindo significamente os impactos ambientais causados pela erosão hídrica.

Palavras-chave: mandioca, preparo do solo, plantas de cobertura do solo, cultivo mínimo.

\section{SUMMARY}

Soil tillage for cassava cropping is tradicionally executed by a number of rowing and tillage which may result in alteration of the physical and chemical soil properties. The use of plants for green cover manure and the use of minimum tillage may contribute to reduction of soil erosion and to facilitate manual harvest. An experiment in eutrophic Oxisol, located in Marechal Cândido Rondon country state of Paraná, Brazil, was carried out to collect information on the physical soil properties, harvest effort strength and cassava productivity. Experimental design was composed by eight treatments with four repetitions on a $2 \times 4$ factorial. Were used two soil tillage (conventional and minimum) and four soil cover types: common vetch (Vicia sativa), black oat (Avena strigosa), common vetch plus black oat and bare soil. The results showed that soil density and resistance to penetration in the $0-10 \mathrm{~cm}$ layer were higher under conventional than under minimum tillage. There were no differences for cassava harvest effort and cassava productivity between soil tillage treatments as well as among types of soil cover. No significant interaction was detected also. It is hypotesized that the results obtained are due to the fact that soil used did not have compaction, the soil had a high degree of natural fertility and there was no water stress during the experiment. Consequenthy, minimum tillage may substitute conventional tillage and reduce costs for cassava cropping.

Key words: cassava, minimum tillage, green manure.

\section{INTRODUÇÃO}

No processo de produção agrícola, há a necessidade de que o solo esteja em condições

\footnotetext{
${ }^{1}$ Engenheiro Agrônomo, Doutor. Professor Adjunto do Departamento de Agronomia da Universidade Estadual do Oeste do Paraná (UNIOESTE). 85960-000 Mal. Cândido Rondon, PR. Autor para correspondência.

${ }^{2}$ Aluno de Graduação, Curso de Agronomia, bolsista de iniciação científica PIBIC-CNPq, UNIOESTE. Recebido para publicação em 14.05.99. Aprovado em 22.03.00
} 
físicas, químicas e biológicas adequadas para o desenvolvimento das plantas cultivadas (GUPTA \& LARSON, 1982). O preparo do solo é uma das operações agrícolas na qual se procura alterar seu estado físico, químico e biológico, de forma a proporcionar melhores condições para o máximo desenvolvimento das plantas cultivadas. Todavia, sob a ação dos agentes climáticos, tais como chuvas e ventos, o solo pode perder parte de seus nutrientes por erosão, quando não é manejado corretamente e não esteja protegido por cobertura vegetal (DERPSCH, 1985).

No sistema convencional, o preparo do solo consiste no revolvimento de camadas superficiais, objetivando incorporar corretivos e fertilizantes, aumentar os espaços porosos e com isso aumentar a permeabilidade e o armazenamento de ar e água, facilitando o crescimento das raízes das plantas (BRAUNAK \& DEXTER, 1989). Além disso, o revolvimento do solo promove o corte e o enterrio das plantas daninhas e auxilia no controle de pragas e patógenos do solo (GADANHA JÚNIOR $\boldsymbol{e} t$ al., 1991). Esse revolvimento é realizado, basicamente, com aração e gradagens, cujo arado efetua o corte, elevação, inversão e queda, com um efeito de esboroamento de fatias de solo denominadas de leivas. A grade complementa esse trabalho, diminuindo o tamanho dos torrões na superfície, além de nivelar o terreno. Entretanto, tal prática pode acarretar sérios problemas com o passar dos anos, principalmente se não for feita com critério.

Revolvendo-se o solo, altera-se a agregação, principalmente das argilas, que retém a maior parte dos nutrientes necessários às plantas, facilitando o seu arraste pela ação da chuva e do vento, causando erosão (WÜRSCHE \& DENARDIN, 1980). Segundo os autores, com a inversão das leivas, enterra-se a cobertura vegetal deixando a superfície do solo exposta aos agentes da erosão e, também, à maior evaporação da água armazenada no solo.

A erosão pode aumentar com o uso excessivo de equipamentos de preparo do solo, sendo que esse incremento será maior se o solo permanecer descoberto no período de maior intensidade de chuva (BENATTI JÚNIOR $\boldsymbol{e} \boldsymbol{t} \boldsymbol{a l}$., 1983).

Outro problema, oriundo do uso excessivo e inadequado de arado e grade, é a compactação do solo nas camadas subsuperficiais (conhecida como pé-de-arado ou pé-de-grade). Essas camadas compactadas tendem a aumentar a erosão, pois dificultam a infiltração da água da chuva, saturando rapidamente o solo, e com isso aumentando o escorrimento superficial da água que arrasta consigo as partículas do solo (CAMARGO, 1983).
O uso de adubação verde e o preparo do solo com o mínimo de mobilização são técnicas agrícolas que podem contribuir para diminuir a perda de água no solo (RYDBERG, 1990), e melhorar suas propriedades físicas, como densidade e resistência à penetração (KAYOMBO \& LAL, 1993; HALL et al., 1994 e DAO, 1996).

A cultura da mandioca representa uma importante fonte de renda para os pequenos agricultores da região oeste do Paraná, especialmente no Município de Marechal Cândido Rondon, PR. Nessa cultura, o preparo do solo é tradicionalmente realizado com uma aração e duas gradagens, o que pode trazer problemas de erosão, compactação e empobrecimento progressivo do solo $\mathrm{e}$, conseqüentemente, diminuir a produtividade com cultivos sucessivos.

Vários pesquisadores (DERPSCH, 1985; MONEGAT, 1991; CALEGARI, 1993) enfatizam a importância da rotação de culturas com o uso de plantas de cobertura do solo no cultivo das espécies vegetais, pois além de cobrirem o solo, diminuindo a ação erosiva da chuva, melhoram sua fertilidade natural, reduzem o ataque de pragas e doenças, que são mais intensos quando o cultivo de uma única espécie é realizado sucessivamente na mesma área.

A manutenção de uma cobertura morta é importante na cultura da mandioca, pois ela protege o solo contra os agentes erosivos (DERPSCH, 1985; CALEGARI, 1993), já que a mandioca apresenta crescimento inicial relativamente lento coincidindo com períodos de chuvas intensas. Além disso, a cultura deve ficar livre da competição com plantas daninhas até no mínimo 60 dias após o plantio (LORENZI \& DIAS, 1993).

Este trabalho foi conduzido com o objetivo de avaliar a produtividade da cultura de mandioca, com manejo do solo pelo método convencional e de cultivo mínimo e em sucessão à aveia ou/e ervilhaca.

\section{MATERIAL E MÉTODOS}

Conduziu-se um experimento a campo com a cultura da mandioca no período de julho de 1997 a junho de 1998, no município de Marechal Cândido Rondon, PR, em um Latossolo Roxo eutrófico de textura argilosa, com as seguintes características na profundidade de $0-20 \mathrm{~cm}$ : $\mathrm{pH}$ em $\mathrm{CaCl}_{2}(0,01 \mathrm{M}) 5,3 ; \mathrm{MO} 38 \mathrm{~g} \mathrm{~kg}^{-1}, \mathrm{P} 11,5 \mathrm{mg} \mathrm{dm}{ }^{-3} ; \mathrm{K}$ $0,61 \mathrm{cmol}_{\mathrm{c}} \mathrm{dm}^{-3} ; \quad 5,48 ; \quad 1,89 ; \quad 0,00 \mathrm{cmol}_{\mathrm{c}} \mathrm{dm}^{-3}$ respectivamente de $\mathrm{Ca}, \mathrm{Mg}$ e $\mathrm{Al}$ trocáveis; $4,96 \mathrm{cmol}_{\mathrm{c}} \mathrm{dm}^{-3}$ de $\mathrm{H}+\mathrm{Al}$ e saturação de bases $61,67 \%$.

Utilizou-se o delineamento experimental de blocos casualizados, com oito tratamentos distribuídos em fatorial 4x2 (quatro tipos de 
cobertura vegetal combinados com dois sistemas de preparo do solo), com 4 repetições, em parcelas de 10 metros de comprimento por 4 metros de largura. Os dois sistemas de preparo do solo foram o preparo convencional com uma aração e duas gradagens e o cultivo mínimo, no que somente a faixa de solo na linha de plantio foi revolvida com um sulcador. Os tratamentos de cobertura vegetal foram: aveia preta, ervilhaca comum, aveia consorciada com ervilhaca e pousio durante o inverno. A produção média de fitomassa das plantas de cobertura de solo estão expostos na tabela 1 .

As máquinas e implementos utilizados para conduzir o experimento foram: um trator com cerca de $60 \mathrm{~kW}$ no motor, um arado de 3 discos com diâmetro de 0,637m (26”), uma grade de discos leve, um sulcador e um pulverizador costal.

As plantas de cobertura do solo foram dessecadas, no início do florescimento, com a aplicação do herbicida Glyphosate na dosagem recomendada. Após a dessecação, foram feitos os preparos de solo correspondentes a cada tratamento, efetuando-se a seguir o plantio de mandioca em quatro sulcos por parcela em fileira simples com espaçamento de $1,00 \mathrm{~m}$ entre fileiras e $0,60 \mathrm{~m}$ entre plantas. Não foi aplicado fertilizante mineral tanto para os adubos verdes quanto para a mandioca. Consideraram-se as fileiras laterais como bordaduras, ficando apenas as duas fileiras centrais como área útil para determinação da produtividade.

$\mathrm{O}$ teor de água no solo foi determinado conforme o método descrito por NOGUEIRA (1971) nas camadas de $0-10 \mathrm{~cm}, 10-20 \mathrm{~cm}$, e 20 $30 \mathrm{~cm}$, aos 90 e 240 dias após o plantio da cultura da mandioca.

Para a avaliação da densidade do solo, seguiu-se o método do torrão impermeabilizado com parafina, enquanto a resistência do solo à penetração (linha e entrelinha da cultura da mandioca) foi obtida através do penetrógrafo de marca

Tabela 1 - Produção de matéria seca das plantas de cobertura do solo para o plantio da mandioca. Resultados médios de oito repetições.

\begin{tabular}{cl}
\hline Cobertura vegetal & Matéria seca $\left(\mathrm{kg} / \mathrm{ha}^{-1}\right)$ \\
\hline Aveia & $2635 \mathrm{~b}^{*}$ \\
Ervilhaca & $2727 \mathrm{~b}$ \\
Aveia e Ervilhaca & $3458 \mathrm{a}$ \\
& \\
\hline
\end{tabular}

* médias seguidas por letras diferentes diferem pelo teste de Tukey a $5 \%$.
SOILCONTROL SC-60. Essas avaliações foram realizadas juntamente com a retirada de amostra do solo para determinar o teor de água.

A colheita da mandioca foi realizada 240 dias após o plantio. Avaliou-se a produtividade, medindo-se o peso de raízes em 28 plantas por parcela e determinou-se a força exigida para $o$ arranquio manual das mesmas, com o uso de um dinamômetro de mola, em 14 plantas por parcela.

Os resultados relativos aos parâmetros avaliados foram submetidos à análise de variância simples, verificando a interação entre os fatores avaliados e as médias comparadas pelo teste Tukey a $5 \%$ de significância.

\section{RESULTADOS E DISCUSSÃO}

Os valores médios dos teores de água, densidade do solo e resistência do solo à penetração em função dos sistemas de preparo do solo nas camadas de 0 a 10,10 a 20 e 20 a $30 \mathrm{~cm}$, aos 90 e 240 dias após o plantio da mandioca, estão mostrados nas tabelas 2 e 3 .

O teor de água, nas três camadas de solo e nas épocas amostradas, não foi influenciado pelo sistema de preparo do solo.

Aos 90 dias após o plantio, a densidade do solo e a resistência do solo à penetração na linha e na entrelinha de plantio diferiram significativamente entre os dois sistemas de preparo do solo, na camada de 0 a $10 \mathrm{~cm}$, o que concorda com HALL et al. (1993) e DAO (1996). Nesta camada, a densidade e a resistência do solo à penetração mostraram-se inferiores com a prática do cultivo mínimo, podendo ser explicado pelo fato de ter sido a camada de atuação do sistema radicular das plantas usadas como adubos verdes e pelo solo estar protegido contra o impacto das gotas das chuvas, pela cobertura morta oriunda dessas plantas. Isso, também, foi verificado nas entrelinhas da cultura de mandioca, para a camada de 10 a $20 \mathrm{~cm}$, onde a resistência do solo à penetração foi inferior nos tratamentos com cultivo mínimo ao se comparar com o preparo convencional, independente das espécies de plantas de cobertura do solo.

$\mathrm{Na}$ colheita da mandioca (240 dias), a análise física do solo mostrou que a resistência do solo à penetração na profundidade de 0 a $10 \mathrm{~cm}$, na entrelinha de plantio, foi significativamente menor nos tratamentos com cultivo mínimo, evidenciando que os efeitos do preparo convencional do solo continuaram durante todo o ciclo de desenvolvimento da cultura da mandioca. 
Tabela 2 - Valores médios dos teores de água, densidade do solo e resistência do solo à penetração em função dos sistemas de preparo do solo nas camadas de 0 a 10,10 a 20 e 20 a $30 \mathrm{~cm}$, aos 90 dias após o plantio da mandioca.

\begin{tabular}{|c|c|c|c|c|}
\hline \multirow{2}{*}{$\begin{array}{l}\text { Sistema de } \\
\text { preparo }\end{array}$} & \multirow{2}{*}{$\begin{array}{c}\text { Teores de } \\
\text { água } \\
(\%)\end{array}$} & \multirow{2}{*}{$\begin{array}{l}\text { Densidade } \\
\text { do solo } \\
\mathrm{g} \mathrm{cm}^{-3}\end{array}$} & \multicolumn{2}{|c|}{ Resistência à penetração $\left(\mathrm{kg} \mathrm{cm}^{-2}\right)$} \\
\hline & & & Linha & Entrelinha \\
\hline & & $0 \mathrm{a} 10 \mathrm{~cm}$ & & \\
\hline Cultivo mínimo & $21,3 \mathrm{a}^{*}$ & $1,19 \mathrm{~b}$ & $14,7 \mathrm{~b}$ & $9,5 \mathrm{~b}$ \\
\hline Preparo convencional & $20,8 \mathrm{a}$ & $1,25 \mathrm{a}$ & $25,2 \mathrm{a}$ & $28,5 \mathrm{a}$ \\
\hline Cultivo mínimo & $23,0 \mathrm{a}$ & $1,16 \mathrm{a}$ & $29,6 \mathrm{a}$ & $26,7 \mathrm{~b}$ \\
\hline Preparo convencional & $22,5 \mathrm{a}$ & $1,19 \mathrm{a}$ & $35,9 \mathrm{a}$ & $35,4 \mathrm{a}$ \\
\hline Cultivo mínimo & $23,1 \mathrm{a}$ & $1,23 a$ & $31,2 \mathrm{a}$ & $30,9 \mathrm{a}$ \\
\hline Preparo convencional & $23,1 \mathrm{a}$ & $1,18 \mathrm{a}$ & $32,9 \mathrm{a}$ & $35,1 \mathrm{a}$ \\
\hline
\end{tabular}

* médias seguidas por letras diferentes, nas colunas, diferem pelo teste de Tukey a 5\%.

A densidade do solo não se alterou com os sistemas de preparo na avaliação realizada aos 240 dias após o plantio nas três camadas avaliadas. No entanto, deve-se salientar que o preparo convencional do solo exerceu efeito de compactação na camada superficial ( 0 a $10 \mathrm{~cm}$ de profundidade) o que é verificado com os dados de resistência à penetração e de densidade do solo avaliados aos 90 dias após o plantio. Conforme relatado por WÜNSCHE \& DENARDIN (1980), o preparo convencional altera a agregação do solo, deixando a superfície descoberta e desprotegida do impacto das gotas da chuva que atingem o solo, desagregando-o, facilitando o arraste de partículas de argilas pela

Tabela 3 - Valores médios dos teores de água, densidade do solo e resistência do solo à penetração em resposta aos sistemas de preparo nas camadas de 0 a 10 , 10 a 20 e de 20 a $30 \mathrm{~cm}$, aos 240 dias após o plantio correspondente ao período de colheita da mandioca.

\begin{tabular}{|c|c|c|c|c|}
\hline \multirow{2}{*}{$\begin{array}{l}\text { Sistema de } \\
\text { Preparo }\end{array}$} & \multirow{2}{*}{$\begin{array}{l}\text { Teores de } \\
\text { água } \\
(\%)\end{array}$} & \multirow{2}{*}{$\begin{array}{l}\text { Densidade } \\
\text { do solo } \\
\mathrm{g} \mathrm{cm}^{-3}\end{array}$} & \multicolumn{2}{|c|}{ Resistência à penetração $\left(\mathrm{kg} \mathrm{cm}^{-2}\right)$} \\
\hline & & & Linha & Entrelinha \\
\hline Cultivo mínimo & $22,0 a^{*}$ & $1,21 \mathrm{a}$ & $5,09 \mathrm{~b}$ & $4,59 \mathrm{~b}$ \\
\hline Preparo convencional & $21,9 \mathrm{a}$ & $1,25 \mathrm{a}$ & $13,29 \mathrm{a}$ & $11,62 \mathrm{a}$ \\
\hline Cultivo mínimo & $23,3 \mathrm{a}$ & $1,17 \mathrm{a}$ & $17,11 \mathrm{~b}$ & $16,34 \mathrm{a}$ \\
\hline Preparo convencional & $23,5 \mathrm{a}$ & $1,19 \mathrm{a}$ & $22,06 a$ & $20,83 a$ \\
\hline Cultivo mínimo & $23,4 \mathrm{a}$ & $1,21 \mathrm{a}$ & $27,01 \mathrm{a}$ & $27,57 \mathrm{a}$ \\
\hline Preparo convencional & $23,7 \mathrm{a}$ & $1,19 \mathrm{a}$ & $32,56 a$ & $29,16 \mathrm{a}$ \\
\hline
\end{tabular}

* Médias seguidas por letras diferentes, nas colunas, diferem pelo teste de Tukey a $5 \%$. água, aumentando o processo erosivo. Além disso, a compactação da camada superficial do solo, verificada no preparo convencional, tende a aumentar a erosão, pois dificulta a infiltração da água da chuva, saturando rapidamente o solo, e com isso aumentando o escorrimento superficial da água que arrasta as partículas do solo (CAMARGO, 1983; MONEGAT, 1991).

A produtividade de raízes e o esforço médio para o arranquio manual das plantas não diferiram significativamente entre os sistemas de preparo do solo e entre os tipos de plantas de cobertura utilizados (Tabela 4), mesmo com a maior compactação superficial observada com o solo preparado no sistema convencional. A ausência de resposta significativa ao uso do cultivo mínimo e plantas de cobertura, deve-se, possivelmente, porque o solo apresenta-se com boas características físicas, alta fertilidade natural e não ocorrência de deficiência hídrica. Possivelmente, em anos com deficiência hídrica, o solo com cultivo mínimo terá maior reserva de água disponível para a cultura (RYDBERG, 1990) e, também, as perdas por evaporação serão menores (BENATTI JUNIOR, $\boldsymbol{e} t$ al. 1983), contribuindo para um melhor desempenho da cultura da mandioca neste sistema de preparo do solo.

A erosão é considerada um dos principais fatores de degradação do solo no Oeste do Paraná, resultando em decréscimo da produtividade. A cultura da mandioca, em função da arquitetura foliar e do grande espaçamento entre linhas e entre plantas na linha, oferece reduzida proteção à superfície do solo no período inicial de desenvolvimento, sendo, portanto, altamente suscetível à erosão hídrica, principalmente nos primeiros 30 dias, que pode ser caracterizado como o período crítico em relação à erosão. Para o desenvolvimento de um sistema sustentável de produção de mandioca, torna-se imprescindível a utilização de sistemas que contemplem o uso de plantas de cobertura do solo, associados à manutenção dos resíduos culturais sobre a superfície. 
Tabela 4 - Valores médios do esforço para o arranquio manual e da produtividade de raízes de mandioca em função do sistema de preparo do solo e das plantas de cobertura do solo.

\begin{tabular}{|c|c|c|}
\hline Tratamentos & $\begin{array}{l}\text { Esforço arranquio } \\
\qquad\left(\mathrm{kgf}_{\text {planta }}{ }^{-1}\right)\end{array}$ & $\begin{array}{l}\text { Produtividade } \\
\quad\left(\mathrm{kg} \mathrm{ha}^{-1}\right)\end{array}$ \\
\hline & Sistema de preparo & \\
\hline Cultivo Mínimo & $25,87 \mathrm{a}^{*}$ & $10958 \mathrm{a}$ \\
\hline Preparo Convencional & $24,51 \mathrm{a}$ & $9149 a$ \\
\hline Aveia & $\begin{array}{c}\text { Cobertura vegetal } \\
24,27 \mathrm{a}\end{array}$ & $9762 a$ \\
\hline Ervilhaca & $26,03 \mathrm{a}$ & $10476 a$ \\
\hline Aveia + Ervilhaca & $23,86 a$ & $8989 a$ \\
\hline Pousio & $26,61 \mathrm{a}$ & $11012 \mathrm{a}$ \\
\hline
\end{tabular}

* Médias seguidas por letras diferentes, nas colunas, diferem pelo teste Tukey a $5 \%$.

\section{CONCLUSÕES}

O preparo convencional do solo pode ser substituído pelo cultivo mínimo, no plantio da cultura da mandioca, pois a produtividade e o esforço para o arranquio das raízes não se alteraram quando não se revolveu o solo.

A aveia e a ervilhaca podem ser utilizadas como plantas de cobertura do solo no cultivo da mandioca, na região Oeste do Paraná.

\section{REFERÊNCIAS BIBLIOGRÁFICAS}

BENATTI JÚNIOR, R., FRANÇA, G.V., MOREIRA, C.A. Manejo convencional e reduzido em quatro tipos de solos na cultura do milho em São Paulo. Campinas : Fundação Cargill, 1983. 68p.

BRAUNACK, M.V., DEXTER, A.R. Soil aggregation in the seedbed: a review. I . Properties of aggregates and beds of aggregates. Soil \& Tillage Research, Amsterdam, v.14, p.259-279, 1989.

CALEGARI, A. Manejo de adubação verde. In: ENCONTRO NACIONAL DE ROTAÇÃO DE CULTURAS, 2, 1992, Campo Mourão. Anais... Campo Mourão, 1993. p.104-116.
CAMARGO, O.A. Compactação do solo e desenvolvimento de plantas. Campinas : Fundação Cargill, 1983. 44p.

DAO, T.H. Tillage system and crop residue effects on surface compaction of a paleusoll. Agronomy Journal., v.88, p.141$148,1996$.

DERPSCH, R. Adubação verde e rotação de culturas. In: ENCONTRO NACIONAL DE PLANTIO DIRETO, 3, 1985, Ponta Grossa. Anais... Ponta Grossa : Fundação ABC, 1985. p.85-104.

GADANHA JUNIOR, C.D., MOLIN, J.P., COELHO, J.L.D., et al. Máquinas e implementos agrícolas do Brasil. São Paulo : Núcleo Setorial de Informações em Máquinas Agrícola, Fundação de Ciências e Tecnologia do Estado do Rio Grande do Sul e Instituto de Pesquisas Tecnológicas do Estado de São Paulo, 1991. 449p.

GUPTA, S.C., LARSON, W.E. Modeling soil mechanical behavior during tillage. In: AMERICAN SOCIETY OF AGRONOMY. Predicting tillage effects on soil physical properties and processes. Madison : Soil Science Society of America, 1982. p.151-178.

HALL, D.J.M., MEKENZIE, D.C., MACLEOD, D.A., $\boldsymbol{e} \boldsymbol{t} \boldsymbol{a l}$. Amelioration of a Hardsetting Alfisol through deep mouldboard ploughing, gypsum application and double cropping.I. Soil physical and chemical properties. Soil \& Tillage Research., Amsterdam, v.28, p.253-270, 1994.

KAYOMBO, B., LAL, R. Tillage systems and soil compaction in Africa. Soil \& Tillage Research, Amsterdam, v.27, p.35-72, 1993.

LORENZI, J.O., DIAS, C.A.C. Cultura da mandioca: Coordenadoria de Assistência Técnica Integral, 1993. 36p. (Boletim técnico 211).

MONEGAT, C. Plantas de cobertura do solo: Características e manejo em pequenas propriedades. Chapecó: Ed. do Autor, 1991. 337p.

NOGUEIRA, J.B. Ensaios de laboratório de solos: esquema São Carlos : Escola de Engenharia de São Carlos, Universidade de São Paulo, 1971. 140p.

RYDBERG, T. Effects of ploughless tillage and straw incorporation on evaporation. Soil \& Tillage Research, Amsterdam, v.17, p.303-314, 1990.

WÜRSCHE, W., DENARDIN, L.E. Conservação e manejo dos solos - I. Planalto Rio-grandense. Considerações gerais. Circular Técnica Nacional de Pesquisa do Trigo, Passo Fundo, n.2, p.1-20, 1980

Ciência Rural, v. 30, n. 6, 2000. 